\title{
Needle Guiding Robot with Five-Bar Linkage for MR-Guided Thermotherapy of Liver Tumor
}

\author{
Nobuhiko Hata, Futoshi Ohara, Ryuji Hashimoto, Makoto Hashizume, and \\ Takeyoshi Dohi \\ Department of Mechano-Informatics, Graduate School of Information Science and \\ Technology \\ The University of Tokyo, Graduate School of Medical Sciences, Kyushu University \\ \{noby, ohara, hashimoto, dohi\}@atre.t.u-tokyo.ac.jp, \\ mhashi@dem.med.kyushu-u.ac.jp
}

\begin{abstract}
This paper reports the robot for horizontal gap open MRI which controls needle orientation effectively in the vertically limited gantry space. We locate the robot including actuators wholly in MR gantry and puncture needle from side of patient's body. The robot has three Degree-of-Freedom (DOF) and can control needle orientation using five-bar linkage mechanism and gimbal mechanism. Two DOF is to actuate five-bar linkage and the other DOF to make five-bar linkage up and down. In experiments, the accuracy of five-bar linkage was $0.89 \pm 0.15$ $[\mathrm{mm}]$ on a whole average and the accuracy of vertical placement of fivebar linkage was $0.13 \pm 0.02$ [mm]. MR images had insignificant degradation of $19.4 \%$ in attrition rate of $\mathrm{S} / \mathrm{N}$ ratio at the maximum. We conclude that the robot can control needle orientation with high repeatability and high MR compatibility in the limited gantry space.
\end{abstract}

\section{Introduction}

Intraoperative Magnetic Resonance Imaging (MRI) is a promising imaging tool for monitoring and guiding thermal therapy of liver tumor [1]-[3]. In MR-guided thermal therapies, needle-shaped thermal device is percutaneously inserted to the target tumor(s) under MRI guidance and coagulates the cancerous tissues at effector tip under thermal monitoring. Unique imaging capability of MRI is useful for deliniating tumor tissue, locating thermal probe, and monitoring thermal effect by thermal imaging. MRI guided thermal therapies has been getting attention since the introductoin of open MRI in clinical practice. The open MRI allows more unrestricted access to the patient and the operative field than conventional closed-magnet MRI. Up to date, two configurations, i.e. vertical gap scanner and horizontal gap scanner, of open MRI have been in clinical use to assess their feasibilities in thermal therapies with laser, microwave, radiowave, and cryogenic device $[1]-[3]$.

In order to further enhance the capability of MR-guided thermal therapy of liver tumor, two challenges needs to be addressed. First, the targeting should 
be assisted by intuitive and reliable navigation. Second, actual execution of insertion should be assisted by needle holding device, and possively with active guidance by robot. There are several studies reported to achieve these goals by integrating navigation software, MR compatible robot, and MR scanner [4]-6]. Most of the studies reported are designed for use in either vertical gap scanner, or conventional close-magnet scanner.

In this paper, we propose a new MR compatible robot specifically designed for use in horizontal gap MR scanner. Specifically, we propose (1) a robot for percutaneous needle puncture from the side opening of the horizontal gap scanner, and (2) five-bar linkage mechanism and gimbal mechanism to achieve both compactness of the robot, maximum degree-of-freedom, and large work-space for needle localization.

The engineering significance of this paper is that the combination of mechanism proposed is, to the authors' best knowledge, the first original contribution for percutaneous robotic needle insertion in horizontal open MR scanner. The paper is also clinically significant since the proposed robot has potential to prevail and impact clinical practice; unlike previously published MR-compatible robot for vertical gap scanner, our robot can be integrated into horizontal gap MR scanner which has been already widespread in clinical sites.

\section{Methods}

\subsection{System Design}

Fig:1illustrates the system configuration of the needle guiding robot proposed in this study. Total system consisted of four components: horizontal gap open MRI, MR compatible robot, console for imaging, control PC for robot. The horizontal gap open MRI and the console are part of commercially available 0.3T MRI (AIRISII, Hitachi, Tokyo, Japan). Upon initial volume scanning for localization of tumor site(s), images are transferred to the control PC for planning of needle placement. A set of software were developed and implemented on the control PC (CPU: Pentium 4, 2.53 GHz, RAM: 1024 MB) using the 3D Slicer. The 3D Slicer is a surgical simulation and navigation software program, which displays multimodality images three- and two-dimensionally [7, [8]. The 3D Slicer was used in this study to transfer intraoperative images from the scanner and perform tumor segmentation followed by robot control.

Needle guiding robot is placed on the side of the patient to allow horizontal to near-vertical access of the needle to tumor targets in the liver through opening of the Radio Frequency(RF)-coil. The alignment of the robot with respect to the patient (and the targets in the liver) is manual; yet, the needle placement is robotized by three degree-of-freedom (DOF) motion of the needle guiding robot.

\subsection{Five-Bar Linkage Mechanism and Gimgal Mechanism}

The proposed robot has five-bar linkage mechanism and gimbal mechanism, to achieve three DOF motion of the needle placement. The needle guide is held by 


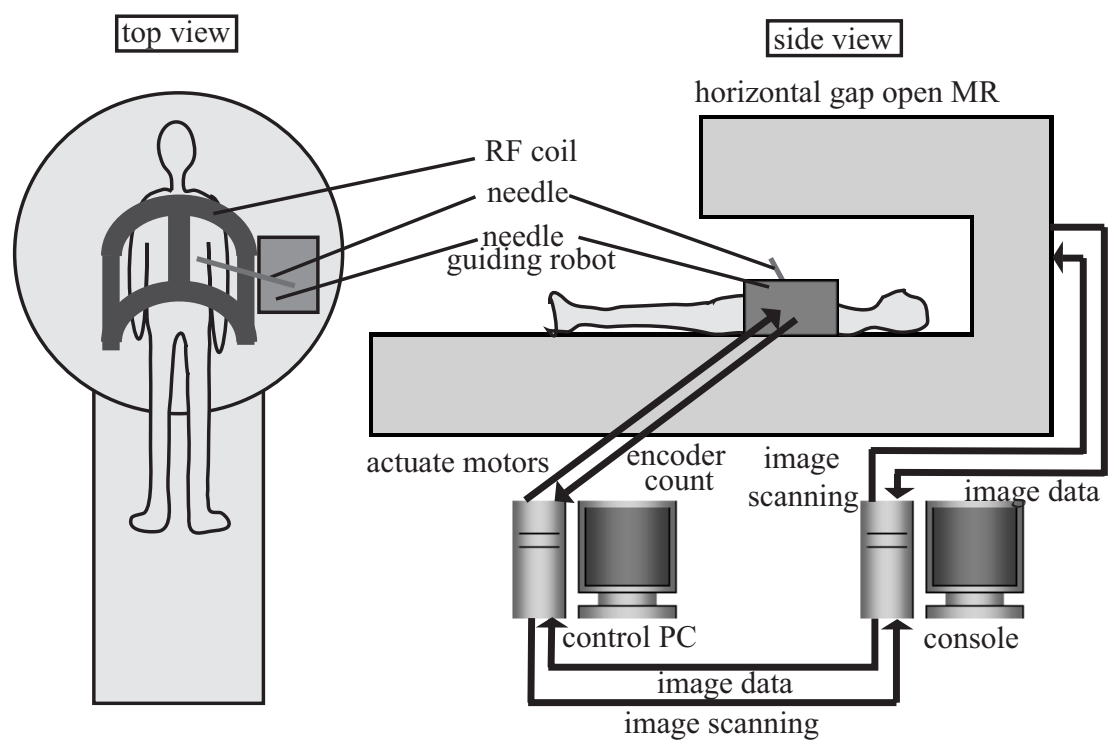

Fig. 1. Schematics illustration of MR compatible needle guiding robot for horizontal gap open MR scanner. Needle guiding robot is placed on the side of the patient enabling horizontal to near-vertical needle placement through opening of the RF-coil.

the top gimbal at the tip of five-bar linkage, and the bottom gimbal at the tip of fixed-bar (Fig 2). Both gimbals enables two rotational motions of the needle guide, though only the bottom gimbal fixes the needle guide to limit the slippage of the needle guide. The five-bar linkage is lifted with respect to the fixed edge unit, to enable vertical rotation of the guide needle.

The five-bar linkage consists of four closed links shown in Fig2. Two links attached to the base stage rotates around the axis on the base and the other two extended links are connected at the tips each other to form closed link. By rotating links at the two 'actuated points' on motor axis, the 'link tip' can be arbitrary placed on horizontal plane formed by the linkages.

The gimbal holds a needle by limiting the freedom-of-motion on two rotation and one slide motion. Specifically, the horizontal rotations of the needle around the gimbal is possible by horizontal motion of five-bar linkage, and the vertical rotation of the needle is possible by lifting the five-bar linkage with respet to the 'fixed edge'. The rotation around the needle axis is prohibited by the fixer on the tail tip.

\subsection{Size Specification}

Based on the design concept mentioned above, we developed a robot shown in (Fig[3). The height of the robot is $170[\mathrm{~mm}]$ at the lowest state and $240[\mathrm{~mm}]$ at the highest state against the height of MR gantry 420 [mm]. Therefore needle 


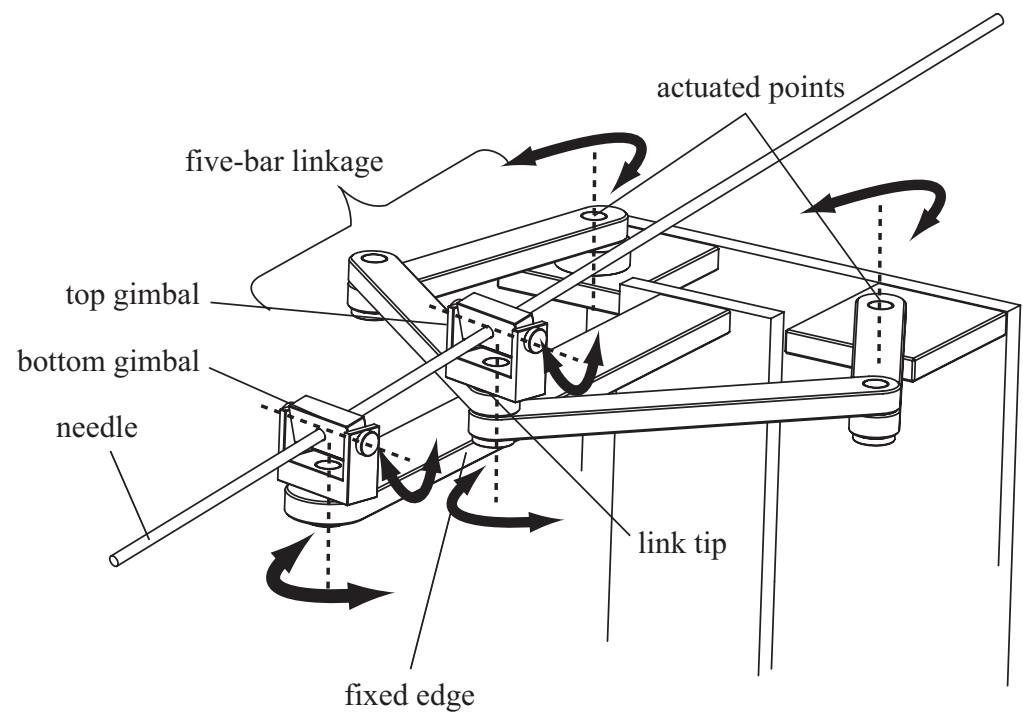

Fig. 2. Five-bar linkage mechanism and gimbal mechanism achieving 3 DOF motion of needle. The needle is held by two gimbals; the top gimbal is attached at the tip of the five-bar linkage manipulator while the bottom one is placed on fixed bar. The needle position and orientation is determined by the in-plane motion of the five-bar linkage.

can tilt up to 36 [degree] against horizontal plane when the robot is in highest state in the gantry, supposing the needle length 300 [mm]. Encoder resolution for motor control was 360/2000 [degree]. Links at the side of 'actuated points' are $75[\mathrm{~mm}]$ long, links at the side of 'link tip' are 95 [mm] long, and diameter between 'actuated points' is 80 [mm]. 'Link tip' can move along superior-toinferior axis of the patient by stroke of $110[\mathrm{~mm}]$, and along left-to-right stroke of $92[\mathrm{~mm}]$. Vertical motion of five-bar linkage is actuated by ultrasonic motor (USR60-E3N, Shinsei kogyo, Tokyo, Japan) and lead screw (stroke; 70 [mm] and resolution; $2[\mu \mathrm{m}])$.

The robot has three passively controlled motion. The first is needle insertion designed to be operated manually by surgeon. The second is vertical motion of the 'fixed edge' with $70[\mathrm{~mm}]$ stroke and the third is whole robot's movement along body axis of the patient. The motion along body axis has stroke of 250 $[\mathrm{mm}]$ to cover a whole liver inside needle's target range.

\subsection{MR Compatible Materials}

Following the detailed report on MR compatibility of robots [?]-7], we carefully chose stainless-, steel-, aluminium-, resin-, ceramic-based parts for translating unit (lead screw and guide rail), five-bar linkage, gimbals, actuators, and the other constitute. 
For lead screw and guide rail in translating unit, we chose stainless steel.Resin was used as a bushing and a nut in translating unit. Ceramic bearing was used as a bushing of lead screw. Five-bar linkage, gimbals and the other constitute consisted of aluminium. Actuators were ultrasonic motors (USR60-E3N and USR30E3N, Shinsei Kogyo, Tokyo, Japan).

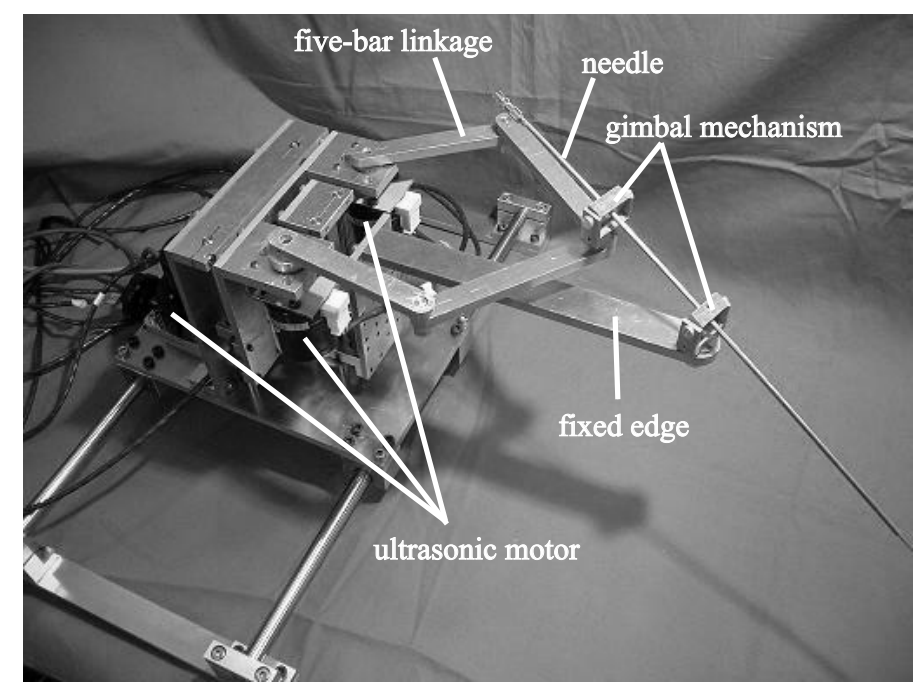

Fig. 3. MR compatible needle holding manipulator

\section{$3 \quad$ Validation Study}

Two sets of experiments were conducted to assess the robot in pre-clinical condition. The first study was to assess the reproducibility of needle placement and the second was to investigate the MR compatibility of the robot. The third set of study was performed with phantom in MR scanner to investigate teh usability of the robot in MRI guided therapy. Measurement of accuracy and reproducibility were tested in dry condition in laboratory while MR compatibility test and phantom study were performed clinical MR scanner. No animal or patient were involved.

\subsection{Accuracy of Needle Tip Placement}

The accuracy and reproducibility of the needle tip placement were assessed in horizontal motion and vertical motion separately. The reproducibility of horizontal motion was tested by setting 2 points as a target and displaced the needle guide tip to the targeted points 10 times in each trial. The motion of the needle 
guide tip was recorded by a digital microscope (VH-7000C, KEYENCE, Osaka, Japan) placed above the robot. The video images from the digital microscope were later processed to digitize the position of the tips with a resolution of 0.07 $[\mathrm{mm}]$. The result of measured standard deviation indicated from 0.07 [mm] to 0.14 [mm].

The accuracy of vertical needle placement was approximated by measuring the accuracy of the base-stage plamement. The position of the base-stage was measured by a laser gauge(LK-080, KEYENCE, Osaka, Japan) placed above the robot. We set lowest state as a starting position and displaced the base stage from $5[\mathrm{~mm}]$ to $70[\mathrm{~mm}]$ with an interval of $5[\mathrm{~mm}]$. The result indicated the accuracy of vertical motion was $0.13 \pm 0.02[\mathrm{~mm}]$ on a whole average. Errors at each sampling point ranged from $0.11[\mathrm{~mm}]$ to $0.14[\mathrm{~mm}]$.

\subsection{MR Compatibility Test}

The second set of assessment was MR-cmpatibility test to analyze the effect of the needle guiding robot on MR imaging.

A polyethylene cylindrical vessel filled with aqueous solution of $\mathrm{NaCl}$ and $\mathrm{MnCl}_{2}$ was used as a phantom. The MR imaging was gradient field echo, a typical imaging sequence for MR-guided thermal therapy, with imaging parameters as follows; TR/TE: 50/20 [ms], FOV: 256 [mm], matrix: 256×128, slice thickness: 10 [mm] flip angle: 90 [degrees].

The images were taken under the following conditions;

Condition 1: Control (phantom only)

Condition 2: The robot installed in the MR scanner and electric power unplugged

Condition 3: The robot installed in the MR scanner actuated (one motor)

Condition 4: The robot installed in the MR sccaner and all axis actuated (linkage actuated).

In order to evaluate the noise, $\mathrm{S} / \mathrm{N}$ ratio was calculated in eace image using the following equation;

$$
S / \text { Nratio }=I_{\text {center }} / S D_{\text {corner }} .
$$

$I_{\text {center }}$ indicates the average intensity of 120 pixel in diameter at the center of the image and $S D_{\text {corner }}$ is the average of standard deviation of the 60 pixel in diameter area at the corner of the image. Imaging and measurement were repeated five time per each of four conditions. Illustrative image for each condition and the measured results are shown in Fig 4 Image degradation in $\mathrm{S} / \mathrm{N}$ ratio was maximum $19.4 \%$ when all theme actuators were in motion.

\subsection{Phantom Study in Clinical MR Scanner}

We conducted a set of phantom studies to evaluate the feasibility of the robot in near clinical setting with MR scanner. We also measured the succsess ratio of needle targetting with pseudo tumors (vitamin E capsules $8[\mathrm{~mm}]$ in diameter) placed in an agar phantom $(150[\mathrm{~mm}] \times 250[\mathrm{~mm}] \times 100[\mathrm{~mm}])$. Four capsules 


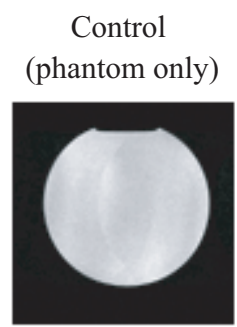

S/N ratio: 305.2 robot installed (motor unplugged)

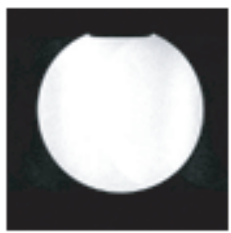

S/N ratio: 300.9 robot actuated (one motor)

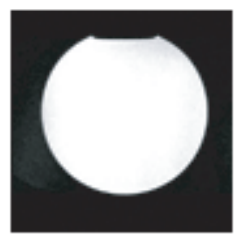

S/N ratio: 275.0 robot actuated (link actuated)

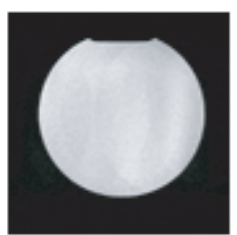

S/N ratio: 245.9

Fig. 4. $\mathrm{MR}$ phantom image and $\mathrm{S} / \mathrm{N}$ ratio from the $\mathrm{MR}$ compatibility test. Imaging condition are given on the top of each image.

were placed in the phantom with known geometical correlation, while setting the one capsule as reference point and the other three as pseudo tumors. The needle tip (14-gauge) was first placed at the reference point in the beginning of each trial, and then repositioned to one of the pseudo tumors by the robot. 10 trials were repeated per each target tumor and number of successful hit were counted by observing the MRI. Fig 5 shows an illustrative MR images of a target in the phantom and the same target hit by the needle. We could successfully hit the tumor in all trials.
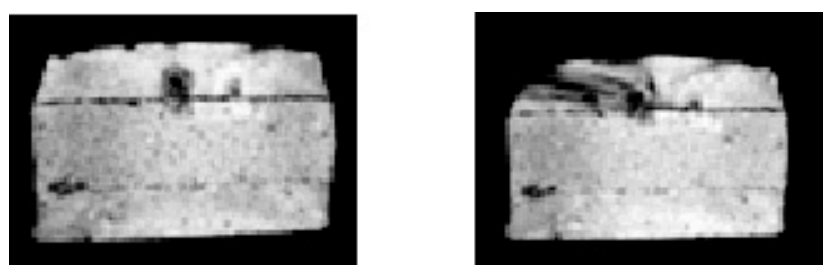

Fig. 5. MR phantom images of needle insertion test. Left image is the image before needle insertion and right image is the image after the needle was inserted.

\section{Discussion}

We developed a robot specifically designed for use in horizontal gap open MRI and tested the feasibility in near clinical setting. The robot has three DOF and can control needle orientation using five-bar linkage mechanism and gimbal mechanism. The size of the robot is 170 [mm] in the lowest state and $240[\mathrm{~mm}]$ in the highest state to actuate needle in the limited gantry space of MR. And 
five-bar linkage tip can cover $110[\mathrm{~mm}] \times 92[\mathrm{~mm}] \times 70[\mathrm{~mm}]$ volume covering nomal sized liver.

In reproducibility experiment of horizontal needle placement, the standard deviation indicated from $0.07[\mathrm{~mm}]$ to $0.14[\mathrm{~mm}]$. We conclude the robot has high reproducibility in the horizontal placement of needle guide.In accuracy and reproducibility experiment of the vertical needle placement, the result indicated $0.13[\mathrm{~mm}] \pm 0.02[\mathrm{~mm}]$. We could achieve high accuracy and reproducibility in the vertical needle placement.

In MR compatibility test, the degradation of $\mathrm{S} / \mathrm{N}$ ratio in each condition was insignificant. The maximum degradation was $19.4 \%$. This result indicates high MR compatibility of the robot and the usability of the robot in MR scanner.

In phantom study in MR scanner, we could successfully hit the pseudo tumors in all trials by observing the MRI. This result indicates that the robot has ability of targetting and hitting the tumor in near clinical setting. Our future work includes development of control theory to hit targets localized in intraoperative MR images, which leads to evalution of the robot in more realistic surgical setting.

Acknowledgement. This study was funded in part by NEDO of Japan. The authors thank Mr. Watanabe of Hitachi Medico for his advice and support on MR imaging.

\section{References}

1. Kettenbach J, Silverman SG, Hata N, et al. Monitoring and visualization techniques for MR-guided laser ablations in an open MR system. J Magn Reson Imaging 1998;8:933-943

2. Morikawa S, Inubushi T, Kurumi Y, et al. MR-guided microwave thermocoagulation therapy of liver tumors: initial clinical experiences using a $0.5 \mathrm{~T}$ open MR system. J Magn Reson Imaging 2002;16:576-583

3. Silverman SG, Tuncali K, Adams DF, et al. MR imaging-guided percutaneous cryotherapy of liver tumors: initial experience. Radiology 2000;217:657-664

4. Masamune K, Kobayashi E, Masutani Y, et al. Development of an MRI-compatible needle insertion manipulator for stereotactic neurosurgery. J Image Guid Surg $1995 ; 1: 242-248$

5. Chinzei K, Miller K. Towards MRI guided surgical manipulator. Med Sci Monit 2001;7:153-163

6. Krieger A, Susil R C, Fichtinger G, et al. Design of A Novel MRI Compatible Manipulator for Image Guided Prostagte Intervention. International Conference of Robotics \& Automation -ICRA 2004 2004;377-382

7. Gering DT, Nabavi A, Kikinis R, et al. An integrated visualization system for surgical planning and guidace using image fusion and an open MR. J Magn Imaging 2001;13:967-975

8. Hata N, Jinzaki M, Kacher D, et al. MR imaging-guided prostate biopsy with surgical navigation software: device validation and feasibility. Radiology 2001;220:263-268 